\title{
Origin of the methylene bonds in poly[2-methoxy-5-(2'-ethyl-hexyloxy)-1,4- phenylenevinylene] prepared according to Gilch's method: novel applications
}

\author{
King-Fu Lin,* Yang-Liang Fan and Hsuan-Liang Chow \\ Department of Materials Science and Engineering, National Taiwan University, Taipei, Taiwan 10617, Republic of China
}

\begin{abstract}
Impurities containing methylene bridges between 2-((2'-ethylhexyl)oxy $)-5-m e t h o x y-b e n z e n e ~ m o l e c u l e s$ are inevitably formed during the synthesis of 1,4-bis(chloromethyl)-2-((2'-ethylhexyl)oxy)-5-methoxy-benzene, the monomer used in the preparation of poly[2-methoxy-5-(2'-ethyl-hexyloxy)-1,4-phenylenevinylene] (MEHPPV), but they can be removed by double recrystallization of the monomer prior to polymerization. When impurities containing methylene bridges participate in a Gilch polymerization, the methylene bonds formed in the main chains are prone to break at $200^{\circ} \mathrm{C}$, that is, at least $150^{\circ} \mathrm{C}$ below the major degradation temperature of defect-free MEH-PPV. Interestingly, the thermal treatment used to break the methylene bonds present reduces the chain aggregation of MEH-PPV during film formation and induces its blends with poly(2,3-diphenyl-5-octylp-phenylene-vinylene) (DPO-PPV) to form a morphology similar to that of block copolymers. Both significantly enhance the luminescence properties.

(C) 2006 Society of Chemical Industry
\end{abstract}

Keywords: conjugated polymers; luminescence; poly[2-methoxy-5-(2'-ethyl-hexyloxy)-1,4-phenylenevinylene] (MEH-PPV); light-emitting diodes (LED)

\section{INTRODUCTION}

With the merits of low turn-on voltage, good solubility and high electroluminescent (EL) efficiency, poly[2methoxy-5-(2'-ethyl-hexyloxy)-1,4-phenylenevinylene] (MEH-PPV) has attracted much attention for its potential applications in polymer light-emitting diodes (PLEDs). ${ }^{1-7}$ The preparation of MEH-PPV is generally carried out through dehydrohalogenation of 1,4-bis(halo-methyl)-2-((2'-ethyl-hexyl)oxy)5-methoxy-benzene) monomer, a method that was originally proposed by Gilch and Wheelwright. ${ }^{8}$ Many researchers have attempted to improve its EL performance by thermal annealing above its glass transition temperature in order to reduce the imperfections of the film..$^{9,10}$

Recently, PPV derivatives prepared via Gilch's route were reported to have some structural defects, such as tolane-bisbenzyl (TBB) moieties, ${ }^{11}$ non-eliminated groups, ${ }^{12}$ and aldehyde. ${ }^{12}$ The presence of TBB in MEH-PPV was shown to be detrimental to the lifetime of the prepared LEDs. ${ }^{13,14}$ Lately, we discovered the presence of methylene defects (or bonds) in $\mathrm{MEH}$ PPV chains - defects that are prone to break at $200^{\circ} \mathrm{C}$, at least $150^{\circ} \mathrm{C}$ lower than the major degradation temperature of defect-free MEH-PPV. This bond has been mistakenly assigned to the cis defect in our previous studies because of the similarity between their ${ }^{1} \mathrm{H}-\mathrm{NMR}$ spectra. ${ }^{15}$ Through thermal annealing of MEH-PPV in order to break the methylene bonds at $200^{\circ} \mathrm{C}$, the extent of chain aggregation was significantly reduced, so that the maximum quantum yield of photoluminescence (PL) was increased to six times that of untreated MEH-PPV. ${ }^{16}$

Moreover, as MEH-PPV containing methylene bonds was blended with poly(2,3-diphenyl-5-octyl$p$-phenylenevinylene) (DPO-PPV) and then submitted to a thermal treatment at $200^{\circ} \mathrm{C}$ for $2 \mathrm{~h}$, the broken MEH-PPV chain segments were able to chemically bond to the DPO-PPV chains by transvinylization. ${ }^{17,18}$ This chemical bonding turned the immiscible blend of polymers into a morphology similar to that of block copolymers. MEH-PPV has a solubility parameter $\delta=20.38\left(\mathrm{~J} \mathrm{~cm}^{-3}\right)^{1 / 2}$, whereas DPOPPV has $\delta=22.93\left(\mathrm{~J} \mathrm{~cm}^{-3}\right)^{1 / 2}$, estimated by the group contribution method. These two polymers are basically immiscible and toluene $\left(\delta=18.21\left(\mathrm{~J} \mathrm{~cm}^{-3}\right)^{1 / 2}\right)$ is a better solvent for MEH-PPV than for DPO-PPV. As toluene was used as a co-solvent for MEH-PPV/DPOPPV polyblend to spin-cast a film, the DPO-PPV phase formed a domain in the upper layer, which dispersed into the more soluble MEH-PPV-rich phase in the wetting layer. Because both the lowest unoccupied molecular orbital (LUMO) and highest occupied molecular orbital (HOMO) levels of MEH-PPV in

\footnotetext{
* Correspondence to: King-Fu Lin, Department of Materials Science and Engineering, National Taiwan University, Taipei, Taiwan 10617, Republic of China E-mail: kflin@ccms.ntu.edu.tw

Contract/grant sponsor: National Science Council of Taiwan, Republic of China; contract/grant number: NSC93-ET-7-002-009-ET Contract/grant sponsor: National Economic Ministry of Taiwan, Republic of China; contract/grant number: 92-EC-17-A-08-S1-0015 (Received 30 November 2005; revised version received 18 January 2006; accepted 31 January 2006)

Published online 16 May 2006; DOI: 10.1002/pi.2043 
the wetting layer are higher than those of DPOPPV in the upper layer, the vertically segregated morphology fits the category of so-called 'type II' heterojunction. ${ }^{19-21}$ As a result, the turn-on voltage of the polymer light-emitting diodes (PLEDs) prepared by the thermally treated polyblend decreased to $\sim 0.6 \mathrm{~V}$, and their EL emission intensities and quantum efficiencies increased to about four times those of the untreated polyblend. ${ }^{17,18}$

In this contribution, we will describe how the methylene bonds form in the MEH-PPV chains and their degradation behavior. Novel applications to enhance the luminescence properties of $\mathrm{MEH}$ PPV and its blends with DPO-PPV through thermal treatment were also elucidated.

\section{EXPERIMENTAL Materials}

All reagents were commercially available and used as received unless otherwise stated. Tetrahydrofuran (THF) was freshly distilled over sodium before it was used. All the reactions were carried out under nitrogen atmosphere. The monomer synthesis and polymerization of DPO-PPV can be found elsewhere. ${ }^{6}$ The monomer used to prepare $\mathrm{MEH}$ PPV, 1,4-bis(chloromethyl)-2-((2'-ethylhexyl)oxy)-5methoxy-benzene, was synthesized according to Ref. 22 with some modifications, which are detailed as follows.

Synthesis of 2-((2'-ethylhexyl)oxy)-5-methoxy-benzene $e^{22}$ A mixture of 4-methoxyphenol $(31 \mathrm{~g}, 0.25 \mathrm{~mol})$ and $\mathrm{KOH}(19.8 \mathrm{~g}, 0.35 \mathrm{~mol})$ in methanol was refluxed for $0.5 \mathrm{~h}$. After cooling to room temperature, 2-ethylhexyl bromide ( $53 \mathrm{~g}, 0.275 \mathrm{~mol}$ ) was added dropwise and further refluxed for $48 \mathrm{~h}$ until the brownish solution turned light yellow. After the methanol had been removed with a rotary evaporator, the remaining mixture was combined with $250 \mathrm{~mL}$ of ether, washed several times with water, and dried over $\mathrm{MgSO}_{4}$. After removing the solvent, $30.5 \mathrm{~g}$ (51.7\% yield) of clear liquid product was obtained.

Synthesis of 1,4-bis (chloromethyl)-2-((2'-ethyl

hexyl)oxy)-5-methoxy-benzene

The compound $(10.62 \mathrm{~g}, 0.045 \mathrm{~mol})$ prepared above was dissolved in $60 \mathrm{~mL}$ dioxane. While the resulting solution was cooled down to $0^{\circ} \mathrm{C}, 45 \mathrm{~mL}$ of concentrated $\mathrm{HCl}$ and $35 \mathrm{~mL}$ of $39 \%$ aqueous formalin solution were added. The solution was further saturated with $\mathrm{HCl}$ by bubbling gaseous $\mathrm{HCl}$ for $20 \mathrm{~min}$ before warming up to room temperature. Afterwards, the mixture was stirred at room temperature for 3 days and then refluxed for $3.5 \mathrm{~h}$. After cooling, concentrating, and storing the reaction mixture in a refrigerator overnight, a precipitate of light-yellow crude product was obtained. This crude product was dissolved in a minimum amount of warm hexane and precipitated with methanol again to afford a white crystalline product $(11.5 \mathrm{~g}, \sim 76 \%$ yield). To distinguish this product (which contained a small amount of impurities having methylene bridges between 2-((2'-ethylhexyl)oxy)-5-methoxy-benzene)s from the further purified monomers, we designated it as MEH-PPV-monomer I. To remove the impurities, further purification was done by dissolving $1 \mathrm{~g}$ of the above product in $50 \mathrm{~mL}$ of isopropanol at refluxing temperature $\left(\sim 85^{\circ} \mathrm{C}\right)$. After complete dissolution, the solution was maintained at $80^{\circ} \mathrm{C}$ for $0.5 \mathrm{~h}$ and then cooled down to room temperature, and a white crystalline product precipitated. After filtration, it was redissolved in chloroform and precipitated with methanol again to obtain MEH-PPV-monomer II $(0.3 \mathrm{~g}, \sim 30 \%$ yield $)$.

\section{Polymerization}

Polymerization of MEH-PPV and DPO-PPV monomers were carried out by the Gilch route. ${ }^{17,18}$ In general, $2 \mathrm{~g}$ of the monomer was first dissolved in $100 \mathrm{~mL}$ of THF. Then, $\sim 4.5$ equiv. of potassium tertbutoxide (Lancaster) dissolved in $150 \mathrm{~mL}$ of THF was added slowly to the stirred monomer solution under nitrogen atmosphere. After complete addition of the base, the reaction proceeded with stirring at room temperature $\left(\sim 25^{\circ} \mathrm{C}\right)$ for a further 3 days. A large quantity of THF was used to prevent the formation of a gel in the polymerization system. At the end of the reaction, the solution was poured into rapidly stirred methanol. The precipitate was then collected by filtration. The resulting polymer was obtained after drying in vacuo. MEH-PPVs resulting from monomers I and II are designated MEH-PPV(I) and MEH-PPV(II), respectively. The molecular weights of MEH-PPVs and DPO-PPVs were determined by gel permeation chromatography (GPC) (Table 1).

\section{Blending and thermal treatment}

MEH-PPV/DPO-PPV blends in 1:1 weight ratio were prepared by dissolving the individual polymers in $\mathrm{THF}$, mixing the two solutions thoroughly, and then drying in vacuo. For thermal treatment, $\sim 0.1 \mathrm{~g}$ of the dried blend was tightly wrapped with aluminum foil to prevent contact with air. The thermal treatment was conducted at $200^{\circ} \mathrm{C}$ for $2 \mathrm{~h}$ under vacuum. The thermally treated samples were then dissolved in $50 \mathrm{~mL}$ THF to remove the undissolved portion by filtration through a $0.4 \mu \mathrm{m}$ filter. After precipitation from the solution by addition of methanol, the

Table 1. Molecular weight and polydispersity of MEH-PPV(I), MEH-PPV(II) and DPO-PPVa

\begin{tabular}{lccc}
\hline Sample & $M_{\mathrm{w}}\left(\mathrm{gmol}^{-1}\right)$ & $M_{\mathrm{n}}\left(\mathrm{gmol}^{-1}\right)$ & $M_{\mathrm{w}} / M_{\mathrm{n}}$ \\
\hline MEH-PPV(I) & 280000 & 43000 & 6.51 \\
MEH-PPV(II) & 333000 & 42900 & 7.76 \\
DPO-PPV & 281000 & 72500 & 3.87 \\
\hline
\end{tabular}

${ }^{a} M_{\mathrm{w}}$, weight-average molecular weight; $M_{\mathrm{n}}$, number-average molecular weight. 
thermally treated polyblend was obtained through filtration and drying in vacuo.

\section{Characterization}

Gel permeation chromatography measurements on $\mathrm{MEH}-\mathrm{PPV}$ and DPO-PPV were carried out at $40^{\circ} \mathrm{C}$ with a Testhigh series III pump and a Testhigh UV detector. One PhenolGEL 550A column and two PhenolGEL MXL columns in series were used with THF as the mobile phase $\left(1 \mathrm{~mL} \mathrm{~min}^{-1}\right)$. The molecular weights were estimated by reference to polystyrene standards with a narrow molecular weight distribution. ${ }^{1} \mathrm{H}$ - and ${ }^{13} \mathrm{C}-\mathrm{NMR}$ spectra of all the samples were recorded on a Bruker Avance 500 spectrometer at frequencies of $500 \mathrm{MHz}$ for ${ }^{1} \mathrm{H}$ and $125 \mathrm{MHz}$ for ${ }^{13} \mathrm{C}$. All spectra were obtained in $\mathrm{CDCl}_{3}$ at room temperature. Chemical shifts refer to tetramethylsilane for ${ }^{1} \mathrm{H}$ and to chloroform- $\mathrm{d}_{1}$ for ${ }^{13} \mathrm{C}$. Thermogravimetric analysis (TGA) of the polymer samples was conducted in a TA model TGA51 thermogravimetric analyzer at a heating rate of $10^{\circ} \mathrm{C} \mathrm{min}^{-1}$ under nitrogen.

The photoluminescence spectrum was recorded on a SPEX 1403 fluorescence spectrophotometer using a xenon lamp as an excitation source. Two-photon excitation microscopy of thermally treated polyblends in film form was carried out with a Leica TCS SP2 confocal spectral microscope imaging system. The wavelength of excitation source was $488 \mathrm{~nm}$, whereas that of the emitting light was collected in the range $500 \sim 700 \mathrm{~nm}$. Film samples were prepared by dissolving the thermally treated polyblend in toluene at room temperature, and then spin-casting onto a glass plate. The preparation procedure of PLED device has been given elsewhere. ${ }^{16}$ The EL spectrum of thermally treated polyblend was recorded on a SPEX1403 fluorescence spectrophotometer at a forward bias of $15 \mathrm{~V}$.

\section{RESULTS AND DISCUSSION}

\section{Origin of the methylene bonds}

Figure 1 shows the ${ }^{1} \mathrm{H}-\mathrm{NMR}$ spectrum of $\mathrm{MEH}-$ PPV-monomer I. Interestingly, a tiny split peak appeared at $\delta=6.6 \sim 6.7 \mathrm{ppm}$, which can only be observed by magnification of the spectrum as shown in the top of the figure. Using column chromatography (silica gel, hexane:ethylacetate = 90:10 by volume) to separate this species from monomer I, and then measuring its heteronuclear multiple quantum correlation (HMQC) spectrum, yielded the result shown in Fig. 2(a). By referring
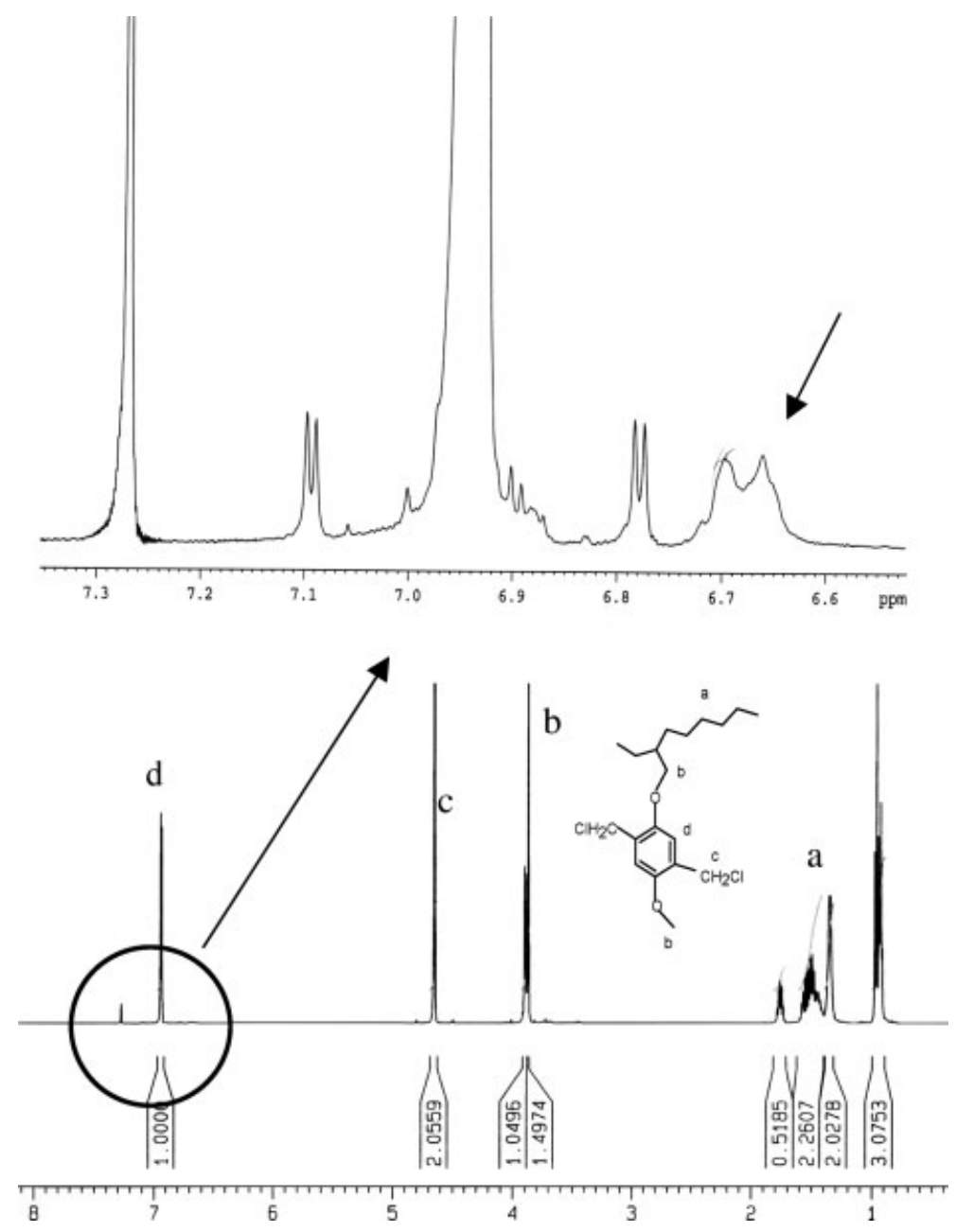

Figure 1. ${ }^{1} \mathrm{H}-\mathrm{NMR}$ spectrum of MEH-PPV-monomer I. Top: Magnified spectrum of the selected area. 
to the reported chemical shift values of bis(4-methyl2,5-dimethoxyphenyl) methane (Fig. 2(b)) which has a methylene bridge between its 2,5-dimethoxybenzenes, ${ }^{23}$ we confirmed that the impurities in MEH-PPV-monomer I contained a similar methylene bridge in between 2-((2'-ethylhexyl)oxy)-5-methoxybenzenes. The original tiny split peak coupled to the carbons at $\delta=113.4$ and $114 \mathrm{ppm}$ in the HMQC spectrum (Fig. 2(a)) was attributed to the phenylene protons of these 2-((2'-ethylhexyl)oxy)-5-methoxybenzenes. The resonance peak of methylene protons at $\delta=3.9 \mathrm{ppm}$ was also coupled to the carbon at $\delta=29.6 \mathrm{ppm}$. Because the reaction scheme to prepare MEH-PPV monomer is similar to that of phenolformaldehyde (except for its higher content in $\mathrm{HCl}$ ) it ought to have a chance to form the methylene bridge during the early stage of monomer synthesis. The species also carried methylene chloride groups on the other side of 2-((2'-ethylhexyl)oxy)-5-methoxybenzenes as indicated by the presence of the peak at $\delta=4.7 \mathrm{ppm}$ (Fig. 2(a)) so that it performed like a monomer. The impurities reached $\sim 10 \mathrm{~mol} \%$ of the MEH-PPV monomer I as estimated from the ratio of peak area at $\delta=6.6-6.7 \mathrm{ppm}$ to its area plus that at $\delta=6.95 \mathrm{ppm}$ (Fig. 1). However, it could be completely removed through double recrystallization of monomer I. We designated the fully purified monomers as MEH-PPV monomer II.

As the MEH-PPV monomer I was subjected to Gilch polymerization, the tiny peak still appeared at $\delta=6.6-6.7 \mathrm{ppm}$ in the ${ }^{1} \mathrm{H}-\mathrm{NMR}$ spectrum of the resulting MEH-PPV(I) (Fig. 3(a)). However, most of the PPV derivatives prepared via the Gilch route also contain tolane-bisbenzyl (TBB) due to headto-head or tail-to-tail reactions. ${ }^{11}$ The phenylene protons of bisphenzyl groups happen to resonate at $\delta=6.6-6.7 \mathrm{ppm}$, which can be seen in the ${ }^{1} \mathrm{H}-\mathrm{NMR}$ spectrum of MEH-PPV(II) (Fig. 3(b)). Dividing the peak intensity at $\delta=6.6 \sim 6.7 \mathrm{ppm}$ by that at $\delta=$ $7.5 \mathrm{ppm}$ contributed by the trans-vinylene bonds for

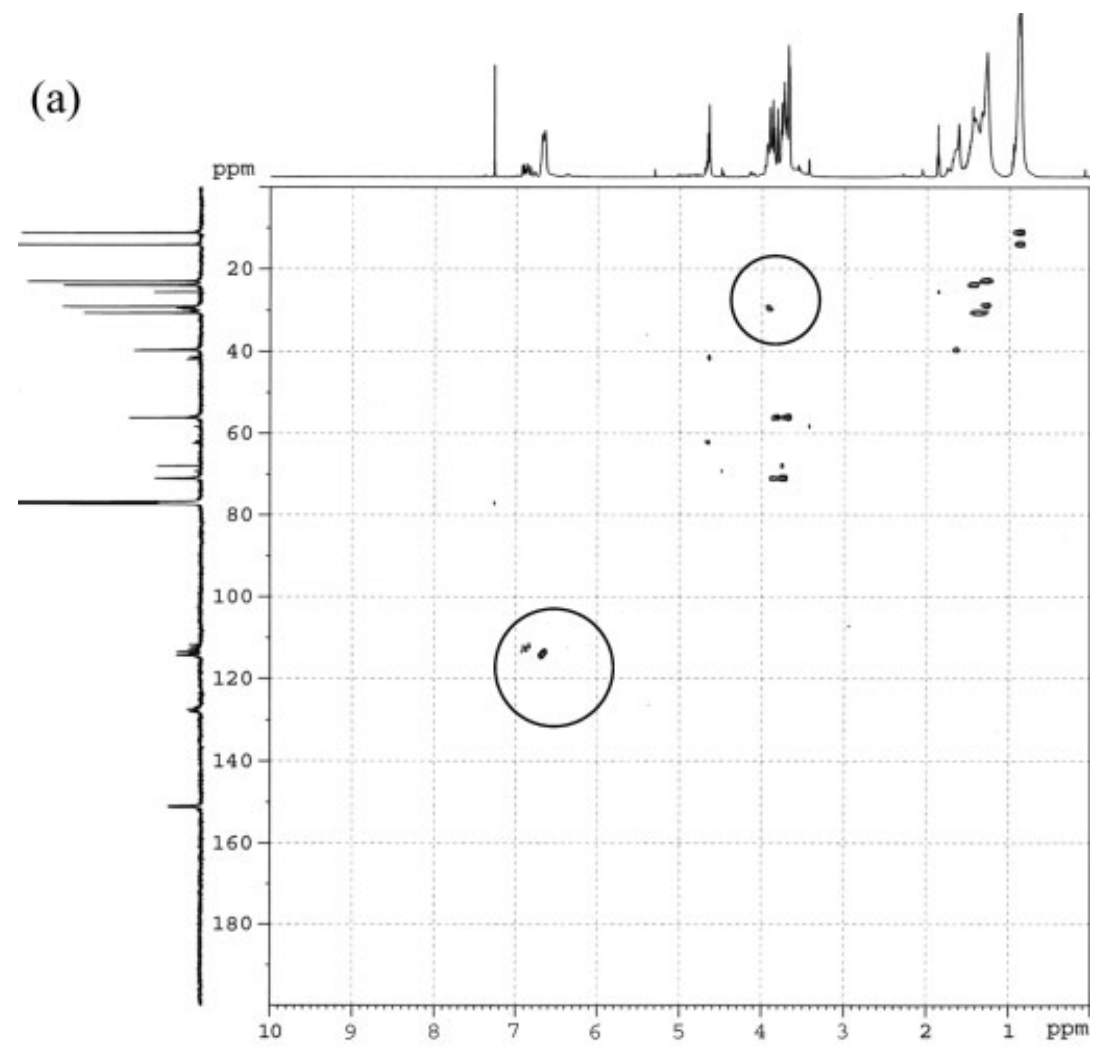

(b)

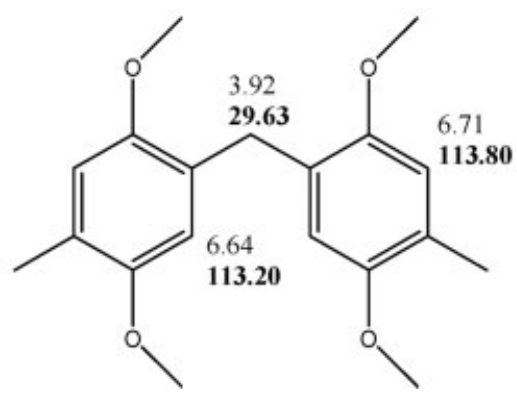

Figure 2. (a) HMQC spectrum of the species containing methylene bridge separated from monomer I through column chromatography and (b) reported chemical shift values of protons and carbons (bold) for the methylene bridge and phenylene groups of bis(4-methyl-2,5-dimethoxyphenyl)methane. ${ }^{23}$ 


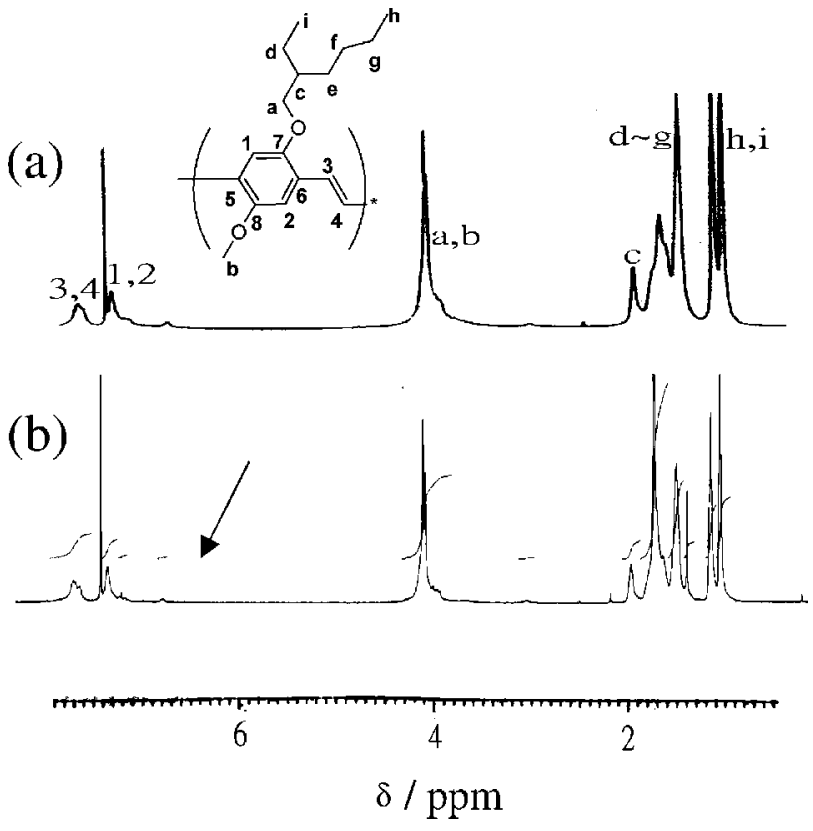

Figure 3. ${ }^{1} \mathrm{H}-\mathrm{NMR}$ spectra of (a) MEH-PPV(I) and (b) MEH-PPV(II).

both polymers, the obtained ratio for the MEH-PPV (I) after subtracting the ratio for the MEH-PPV(II) is also $\sim 10 \%$, which is almost equal to the content of the impurities having a methylene bridge in monomer I. The result indicates that most of the impurities containing methylene bridges participated in the Gilch polymerization.

\section{Degradation of methylene bonds and its novel applications}

It is generally agreed that cis-vinylene protons also resonate at $\delta=6.5-6.8 \mathrm{ppm}$ for the PPV derivatives prepared by the Wittig reactions. ${ }^{24-26}$ In our previous study, we have mistakenly attributed the peak at $\delta=6.6-6.7 \mathrm{ppm}$ for the MEH-PPV prepared from monomer I to the formation of cis-vinylene bonds during Gilch polymerization. ${ }^{15}$ As the resulting polymer was subjected to thermal treatment at $200^{\circ} \mathrm{C}$ for $1-2 \mathrm{~h}$, half of the methylene bonds were broken as indicated by the decrease of the peak at $\delta=6.6-6.7$ ppm. ${ }^{16}$ In this study, we have also found that the MEH-PPV(I) began to degrade at $\sim 200^{\circ} \mathrm{C}$, but that the major degradation took place at $367^{\circ} \mathrm{C}$ (Fig. 4). In contrast to MEH-PPV(I), MEH-PPV(II) started degrading at $\sim 350^{\circ} \mathrm{C}$ but with its major degradation at $395^{\circ} \mathrm{C}$. For MEH-PPV(I), the broken polymer segments during thermal treatment at $200^{\circ} \mathrm{C}$ were highly reactive and tended to chemically bond to the adjacent polymer chains, provoking a reduction of chain aggregation. As a result, the PL quantum efficiency in film form was increased to $\sim 6$ times that of untreated MEH-PPV. ${ }^{16}$

The copolymerization of MEH-PPV and DPO-PPV monomers through the Gilch route tended to form an alternate copolymer with reactivity ratios of $\mathrm{MEH}-$ PPV and DPO-PPV monomers equal to 0.16 and 0.19 , respectively. ${ }^{6}$ As $\mathrm{MEH}-\mathrm{PPV}(\mathrm{I}) / \mathrm{DPO}-\mathrm{PPV}$ blend in

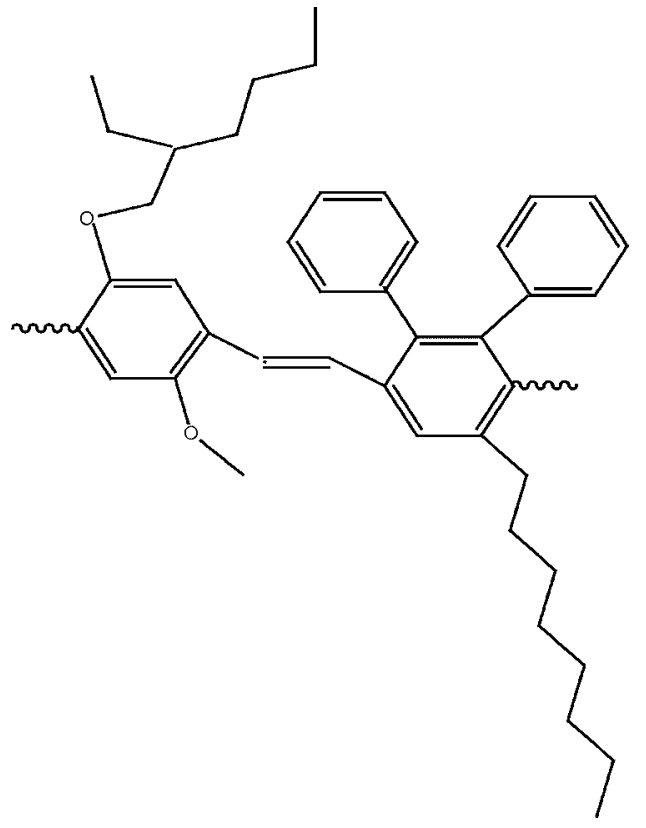

Structure 1.

1:1 weight ratio was subjected to thermal treatment at $200^{\circ} \mathrm{C}$ for $2 \mathrm{~h}$, the broken MEH-PPV chain segments had a tendency to bond to the DPO-PPV chains by trans-vinylization; this is at the origin of the small peak at $\delta=7.35 \mathrm{ppm}$ in ${ }^{1} \mathrm{H}-\mathrm{NMR}$, which stems from the vinylene protons of the moiety (Structure 1), which is the sole vinylene peak for the alternative copolymer prepared from 1:1 molar ratio of $\mathrm{MEH}-$ PPV and DPO-PPV monomers. ${ }^{17,18} \mathrm{MEH}-\mathrm{PPV}$ and DPO-PPV are immiscible. It was also interesting to observe that each DPO-PPV domain (green) of the thermally treated MEH-PPV(I)/DPO-PPV blend was surrounded by a thin layer of red MEH-PPV domains (Fig. 5). As the thermally treated blend was employed as a light-emitting layer for PLED, the turn-on voltage was $0.6 \mathrm{eV}$ - much lower than for that prepared from the untreated polyblend. Its maximum EL intensity was $\sim 2700 \mathrm{~cd} \mathrm{~m}^{-2}$, about four times that of the untreated blend. The EL quantum yield was $2 \mathrm{~cd} \mathrm{~A}^{-1}$, which barely changed with the applied current density, whereas that of the untreated blend decreased from 0.7 to $0.4 \mathrm{~cd} \mathrm{~A}^{-1}$ by increasing the current density. ${ }^{17,18}$

The heterojunction formed between MEH-PPV and DPO-PPV is shown schematically in Fig. 6. MEHPPV acts like a hole-transport polymer and DPO-PPV like an electron-transport polymer. The energy gap of the heterojunction is only $1.98 \mathrm{eV}-$ lower than the band gap of pristine MEH-PPV $(2.14 \mathrm{eV})$. For the thermally treated blend, the maximum emission wavelength of EL spectrum was at $577 \mathrm{~nm}(2.15 \mathrm{eV})$, while that of the PL was at $544 \mathrm{~nm}(2.28 \mathrm{eV})$ as indicated in Fig. 7. The difference is $0.13 \mathrm{eV}$, close to the energy barrier of $0.16 \mathrm{eV}$ between their LUMOs for the excitons to jump from DPOPPV to MEH-PPV in order to cross the LUMO of the heterojunction (Fig. 6). On the contrary, for the untreated MEH-PPV/DPO-PPV blend, the 


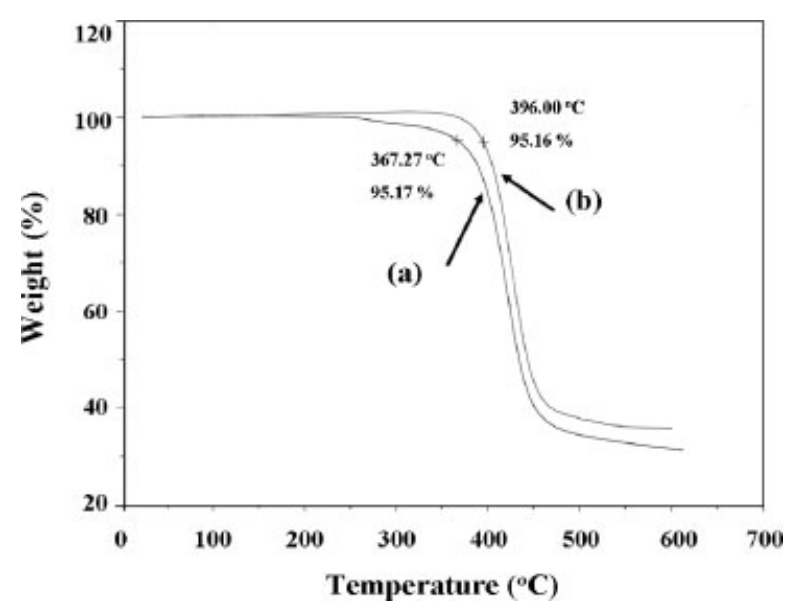

Figure 4. TGA plots of (a) MEH-PPV(I) and (b) MEH-PPV(II).

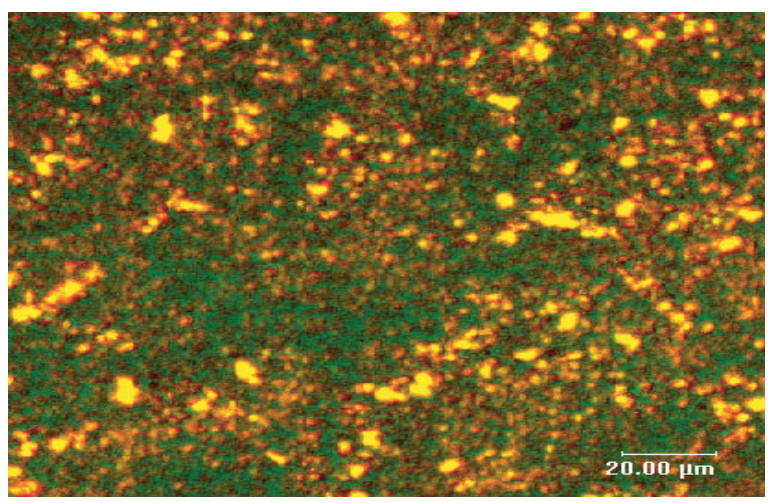

(a)

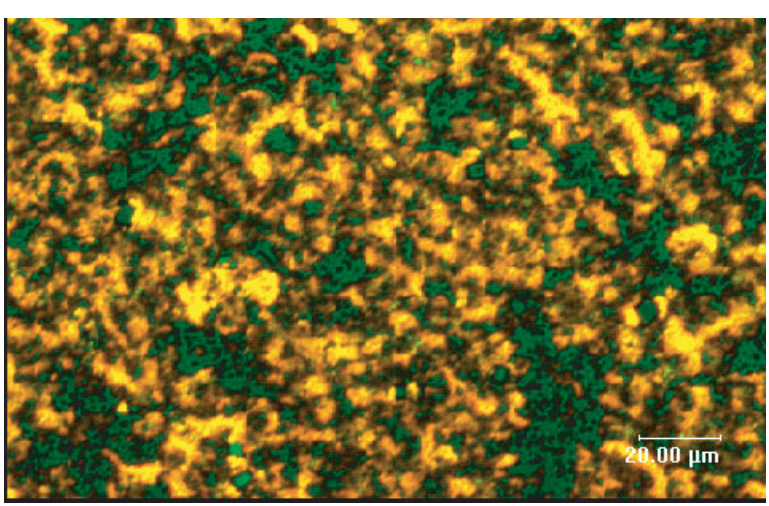

(b)

Figure 5. Photo-excitation micrographs of (a) thermally treated and (b) untreated MEH-PPV(I)/DPO-PPV blend. ${ }^{17,18}$

maximum emission wavelength of PL spectrum is similar to that of the EL spectrum. ${ }^{17,18}$ Therefore, it can be concluded that the thermally treated $\mathrm{MEH}$ PPV(I)/DPO-PPV blend has more heterojunction regions to generate exciplexes than the untreated blend, so that the EL emission is dominated by exciplexes. The EL quantum efficiency was thus significantly increased.

\section{CONCLUSIONS}

We discovered methylene defects in MEH-PPV chains, which could only be eliminated by double

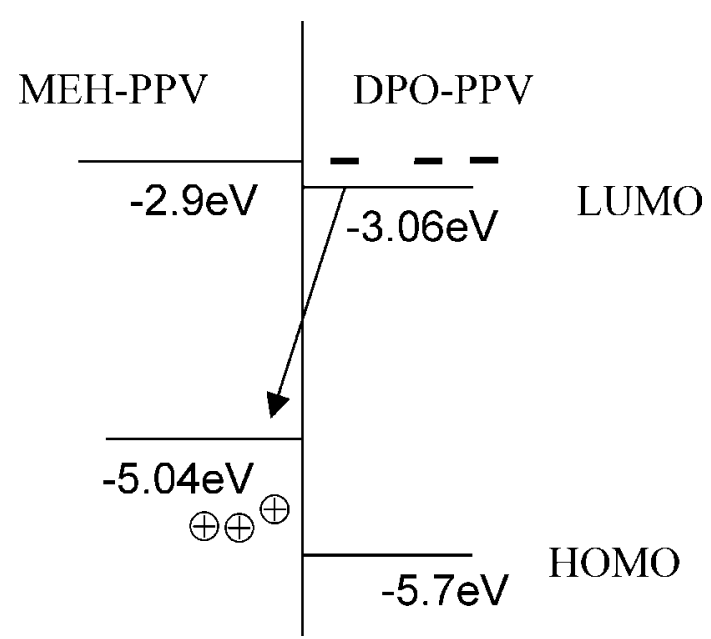

Figure 6. Scheme for heterojunction formed between MEH-PPV and DPO-PPV. Electrons from DPO-PPV and holes from MEH-PPV are trapped and form the exciplexes at the heterojunction during $\mathrm{EL}$ emission.

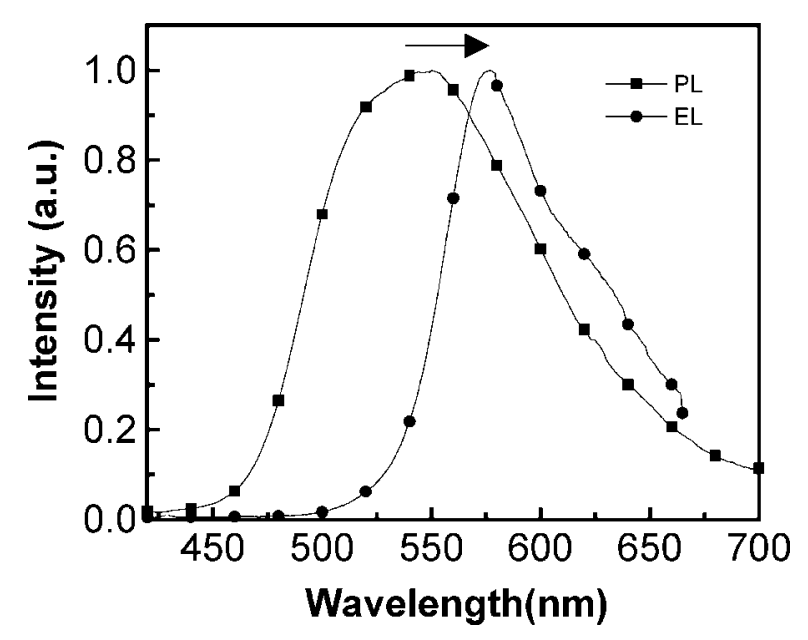

Figure 7. EL and PL spectra of thermally treated MEH-PPV(I)/DPO-PPV blend.

recrystallization of the monomers. Because the methylene bonds are prone to break at $200^{\circ} \mathrm{C}$, a thermal treatment could reduce chain aggregation of $\mathrm{MEH}-$ PPV in film form, and also turn MEH-PPV(I)/DPOPPV blends into block copolymer type structures. Both substantially enhanced the luminescence properties.

\section{ACKNOWLEDGEMENTS}

The authors acknowledge the financial support of the National Science Council of Taiwan, Republic of China, through grant NSC93-ET-7-002-009-ET, and the National Economic Ministry of Taiwan, Republic of China, through grant 92-EC-17-A-08-S1-0015.

\section{REFERENCES}

1 Braun D and Heeger AJ, Appl Phys Lett 58:1982 (1991).

2 Parker ID, $\mathcal{F}$ Appl Phys 75:1656 (1994).

3 Padmanaban G and Ramakrishnan S, f Am Chem Soc 122:2244 (2000). 
4 Collison CJ, Rothberg LJ, Treemaneekarn V and Li Y, Macromolecules 34:2346 (2001).

5 Chen SH, Su AC, Huang YF, Su CH, Peng GY and Chen SA, Macromolecules 35:4229 (2002).

6 Chiu C-C, Lin K-F and Chou H-L, 7 Polym Sci Part A: Polym Chem 41:2180 (2003).

7 Parekh BP, Tangonan AA, Newaz SS, Sanduja SK, Ashraf AQ, Krishnamoorti R, et al, Macromolecules 37:8883 (2004).

8 Gilch HG and Wheelwright WL, 7 Polym Sci Part A: Polym Chem 4:1337 (1966).

9 Lee TW and Park OO, Adv Mater 12:801 (2000).

10 Lee TW, Park OO, Do LM and Zyung T, Synth Met 117:249 (2001).

11 Becker H, Spreitzer H, Ibrom K and Kreuder W, Macromolecules 32:4925 (1999).

12 Roex H, Adriaensens P, Vanderzande D and Gelan J, Macromolecules 36:5613 (2003).

13 Becker H, Gelsen O, Kluge E, Kreuder W, Schenk H and Spreitzer H, Synth Met 111:145 (2000).

14 Becker H, Spreitzer H, Kreuder W, Kluge E, Vestweber H, Schenk H, et al, Synth Met 122:105 (2001)
15 Fan Y-L and Lin K-F, f Polym Sci Part A: Polym Chem 43:2520 (2005).

16 Chou H-L, Lin K-F, Fan Y-L and Wang D-C, 7 Polym Sci Part B: Polym Phys 23:1705 (2005).

17 Chou H-L, Lin K-F and Wang D-C, f Polym Sci Part B: Polym Phys 44:62 (2006).

18 Chou H-L, Lin K-F and Wang D-C, $\mathcal{F}$ Polym Res 13:79 (2006).

19 Corcoran N, Arias AC, Kim JS, MacKenzie JD and Friend RH, Appl Phys Lett 82:299 (2003).

20 Kim J-S, Ho PH, Murphy CE and Friend RH, Macromolecules 37:2861 (2004).

21 Morteani AC, Fiend RH and Silva C, Chem Phys Lett 391:81 (2004).

22 Wudl F and Srdanov G, US Patent 5189136 (1993).

23 Rathore R and Kochi JK, f Org Chem 60:7479 (1995).

24 Pang Y, Li J, Hu B and Karasz FE, Macromolecules 32:3946 (1999).

25 Fan Q-L, Lu S, Lai Y-H, How X-Y and Huang W, Macromolecules 36:6976 (2003).

26 Drury A, Maier S, Rüther M and Blau WJJ, Mater Chem 13:485-490 (2003). 